\title{
Factors Associated with Neonatal Mortality in Bangladesh: A Multilevel Analysis Hemel Das $^{1 *}$ and Nitai Chakraborty ${ }^{2}$ \\ ${ }^{1}$ icddr,b , Mohakhali, Dhaka-1213, Bangladesh \\ ${ }^{2}$ Department of Statistics, Dhaka University, Dhaka-1000, Bangladesh
}

(Received: 21 January 2020; Accepted: 24 February 2021)

\begin{abstract}
This study aims at disentangling the influence of both individual and community level factors on neonatal death in Bangladesh. For analysis purpose, data are extracted from Bangladesh Demographic and Health Survey 2014. Bivariate analysis is used to examine the differentials in neonatal mortality by selected background variables of both levels. As the survey is based on a two-stage stratified sample of households, multilevel logistic regression model is used to analyze the cluster effect and to determine the factors associated with neonatal mortality. Multilevel logistic model confirmed that there exists clustering impact on neonatal death. Moreover, the study has suggested implementation of intervention addressing several individual and community level factors to ameliorate the current neonatal health scenario in Bangladesh.
\end{abstract}

Keywords: Neonatal Mortality, Chi-square test, Multilevel Logistic Regression

\section{Introduction}

Child mortality is considered as a key indicator of overall growth in health sector in any country. The first year for a child is critical period to survive during childhood ${ }^{1}$. The probability of dying a child with 28 days is known as neonatal mortality ${ }^{2}$. A study conducted by UNICEF reported that neonatal death represents 46 per cent of total deaths among children under five ${ }^{3}$ which point out the high concentration of neonatal death instead of a significant reduction in child mortality as an alarming notion for lower-middle income countries like Bangladesh.

The number of neonatal deaths has a decline from 5.1 million in 1990 to 2.6 million in 2016 which is slower than that of post-neonatal under-5 mortality all over the world ${ }^{4}$. As the world is now passing through an era where all countries are in a race of achieving Sustainable Development Goal by 2030 aiming to reduce neonatal mortality to at least as low as 12 per 1,000 live births ${ }^{5}$, the factors influencing neonatal death are needed to be spotted.

In the context of Bangladesh, despite the outstanding progress in reduction of child mortality due to promotion of several life-saving interventions, obstacles like inequalities in terms of access and utilization of health services among the populations still are hindering to achieve the SDG for Bangladesh ${ }^{6}$. According to 2014 BDHS, over the last two decades, the data confirm a steady downward trend in childhood mortality ${ }^{2}$. Between 1993 and 2014 neonatal mortality fell from 52/1000 live births to $28 / 1000$ live births, with newborn deaths now accounting for 61 percent of all under 5 mortality ${ }^{4}$. It reveals that mortality has become increasingly concentrated in the neonates. Though the decline in childhood mortality continues, it would be difficult to achieve the HPNSDP target of 21 neonatal deaths per 1,000 live births for the neonatal mortality rate ${ }^{7}$. Therefore, it is highly demanded to shed light on the reasons that influence neonatal death.
There are many studies that examined the factors affecting neonatal death and they revealed some interesting findings associated with it. Kayode et al. observed through his study that there is a marked impact of both individual and community level factors on neonatal survival in Ghana ${ }^{1}$. Nepaune and Doku investigated the factors influencing neonatal mortality in three levels-individual, community and district and emphasized on the need of proper steps in individual level along with access to health care service to improve the neonatal health in $\mathrm{Nepal}^{8}$. A cross sectional study performed by Titaley et al. also suggested that community, household and individual level factors should be addressed while interventions being implemented ${ }^{9}$.

However, a strand of literature review suggested that both individual and community level variables play a vital role to influence neonatal death. The main aim of this study is to assess the impact of several community level and individual level characteristics on neonatal mortality through a multilevel analysis study.

\section{Methodology}

Data

For the study purpose, data are extracted from 2014 Bangladesh Demographic and Health Survey ${ }^{2}$. The 2014 Bangladesh Demographic and Health Survey (BDHS) is the seventh national-level demographic and health survey ${ }^{2}$ where ever married women aged 15 to 49 are included as respondents. The survey also included a community questionnaire administered during the listing of households to informants in communities around the sample points from which the households were selected.

The survey is based on a two-stage stratified sample of households. In the first stage, 600 EAs were selected with probability proportional to the EA size, with 207 EAs in urban areas and 393 in rural areas. In the second stage of sampling, a systematic sample of 30 households was selected per EA. In accord with this design, 18,000 residential households were selected and completed

"Author for correspondence. e-mail: hemeldas3001@ gmail.com 
interviews were expected from about 18,000 ever-married women age $15-49^{2}$.

\section{Dependent Variable}

In this study, neonatal mortality is considered as dependent variable as children face highest risk of dying in neonate $\operatorname{period}^{10}$. The dependent variable $Y_{i}$ can be defined as follows for $\mathrm{i}^{\text {th }}$ individual-

$Y_{i}=\left\{\begin{array}{l}1, \text { Death occured between } 0-28 \text { days } \\ 0, \text { Death did not occur between } 0-28 \text { days }\end{array}\right.$

\section{Independent Variables}

As one of the main aims of this study is to explore the cluster effect on neonatal death, the independent variables are classified into two groups- individual level and community level.

According to previous literature, several individual level variables are considered in this study. They are Mother's age at birth (less than 15 years, between 15 to 24 years and greater than 24 years), Education level of mother (no education or primary, secondary, higher), Education level of father (no education or primary, secondary, higher), Wealth index (rich, middle/poor), Sex of child (male, female), Number of antenatal visits (no ANC visit, 1 to 3 times, 4 times or more), Delivery by caesarean section (no, yes), Size of child at birth (small, others), Birth order (first birth, second to fourth birth, greater than fourth birth), Preceding birth interval (first birth, less than 2 years, between 2 to 4 years, greater than 4 years), Delivery assistance (unqualified, qualified), Place of delivery (facility, others), Breastfeeding (within 1 hour, later), Postnatal care (PNC) check time (first two days, others), Postnatal care (PNC) by qualified person (no, yes) and Exposer to media (not at all, sometimes/ regular).

The community level variables that are included in this study are- Place of residence (Barisal, Chittagong, Dhaka, Khulna, Rajshahi, Rangpur, Sylhet), Type of region (rural, urban), Electricity availability (yes, no), Telephone service availability (yes, no), NGO activity (yes, no), Availability of fieldworker (yes, no), Presence of qualified doctor (yes, no), Presence of pharmacy (yes, no), Availability of family planning (FP) methods (yes, no).

\section{Statistical Analysis}

To reveal the effect of the variables included in the study on neonatal death, bivariate analysis is employed at first to test the measure of association. Because of sampling design of 2014 BDHS, it may happen that the response of children belonging to same household are correlated. Again, the responses of children among same cluster may also hold some correlation. For this reason, two level multilevel logistic regression model is applied to explore the random effect of cluster along with the adjusted effect of individual and community level of variables on neonatal death.
The specified two-level multilevel logistic regression model is

$$
\begin{gathered}
\operatorname{logit}\left(\pi_{i j} \mid u_{0 j}, e_{i j}\right)=\log \left[\frac{\pi_{i j}}{1-\pi_{i j}}\right] \\
=\beta_{0}+\beta_{1} X_{1 i j}+\beta_{2} X_{2 i j}+\cdots+\beta_{n} X_{n i j}+u_{0 j}+e_{i j}
\end{gathered}
$$

where $\pi_{i j}$ is the probability of death of neonatal $i$, residing in a community $j ; \beta_{0}$ is the $\log$ odds of the intercept; $\beta_{1}, \beta_{2}, \ldots, \beta_{n}$ are the coefficients of individual and community level variables; $X_{1 i j}, X_{2 i j}, X_{3 i j}, \ldots, X_{n i j}$ are individual and community level variables; $u_{0 j}$ is random error at community levels; $e_{i j}$ is random error at individual levels ${ }^{11,12}$.

The random effect of the multilevel model can be estimated by Intra Cluster Correlation (ICC) and Median Odds Ratio (MOR).

The intra-cluster correlation for community level in multilevel logistic model will be

$$
\rho=\frac{\sigma_{u}^{2}}{\left(\sigma_{u}^{2}+3.29\right)}
$$

This correlation measures the proportion of the total variance which is between-cluster ${ }^{11}$.

The MOR depends directly on the community level variance and can be computed with the following formula:

$$
\begin{aligned}
& M O R=\exp \left[\sqrt{\left(2 \times \sigma_{u}^{2}\right)} \times 0.675\right] \\
& \cong \exp \left(0.95 \times \sqrt{\sigma_{u}^{2}}\right)
\end{aligned}
$$

where $\sigma_{u}^{2}$ is the community level variance, and 0.6745 is the $75^{\text {th }}$ centile of the cumulative distribution function of the normal distribution with mean 0 and variance $1^{13}$.

In this study, observations on the dependent variable were collected only from those ever-married woman who gave at least one birth in last three years. Sampling weight is considered in this study for analysis to remove the disparities in response rates among national level and domain level due to non-proportional allocation study design of the survey. After cleaning the cases which have missing information of covariates due to non-response, 4734 observations were available for multivariate analysis. The entire analysis of the study is done by using software SPSS (version 20) and Stata (version 12).

\section{Result}

Table 1 is representing percentage distribution of neonatal death according to demographic and socio-economic characteristics of individual and community level factors included in the study.

Among individual level factors, it is found that education level of mother, education level of father, wealth index, delivery by caesarean section, delivery assistance, breastfeeding, postnatal care time and PNC by qualified 
person has significant impact on neonatal death. It is evident from the result that, and the event of neonatal death is less reported with the increase in education level of both parents. The result shows that there exist a significant negative association between neonatal death and wealth index which follows that the increase of economic condition of a family decrease neonatal death Table 1 also indicates that those who takes caesarean section delivery has lower neonatal death risk $(2.0 \%)$ than those with vaginal delivery $(3.1 \%)$. The neonatal death rate for qualified delivery assistant is 2.2 percent whereas for unqualified delivery assistant the rate is 3.3 percent and the change is statistically significant ( $p$-value $=0.016)$. This study also points out that the child who are given breast milk immediately or within an hour has less chance of neonatal that compared to later. The rate of neonatal mortality is 1.5 percent who took PNC in first two days while 4.2 percent for who did not take PNC in first two days. It is also revealed by the study that if postnatal care is provided by a qualified person then the risk of neonatal mortality is lower (almost 2\%) than those who received postnatal care from unqualified person.

Among community level factors, place of residence, telephone service availability, presence of qualified doctor, presence of pharmacy, availability of family planning methods is found to be significantly associated with neonatal death. Table 1 depicts that neonatal death rate is highest in Sylhet $(5.3 \%)$ while the scenario is opposite in Barisal $(1.8 \%)$. Moreover, that neonatal death rate is extreme where the telephone service is not available $(3.5 \%)$.

Table 1. Percentage distribution of child born in last three years by neonatal death according to demographic and socioeconomic characteristics of individual level and community level factors

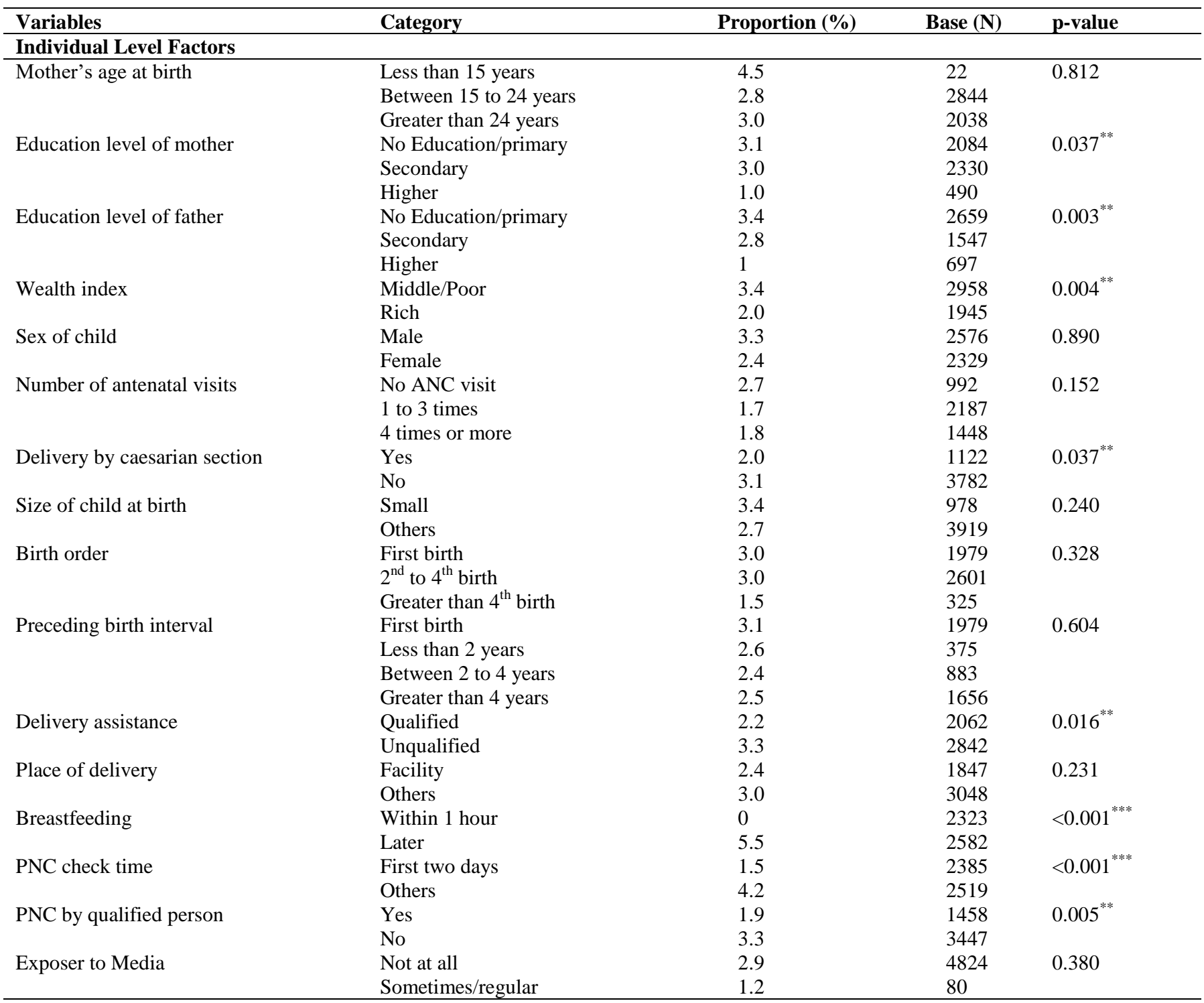


Table 1 continued...

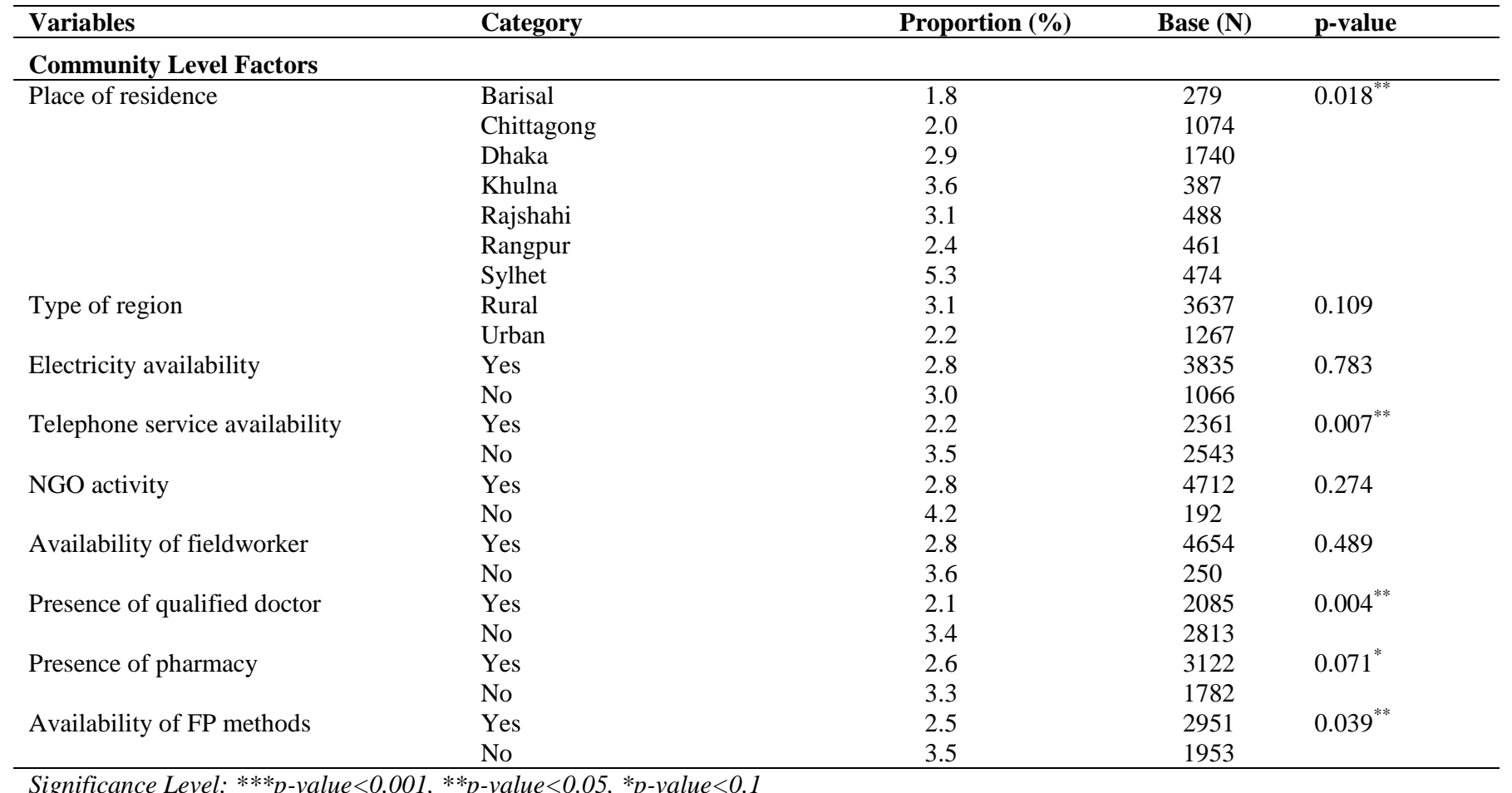

The study reveals that availability of qualified doctor has lowered the percentage of neonatal death $(2.1 \%)$ significantly. Additionally, presence of pharmacy also worked as protective factor from neonatal death. The study also reveals that the clusters where family planning methods are not available are more at risk of neonatal death $(3.5 \%)$.

Table 2 is representing the random effect at community level and fixed effect of several factors on neonatal death obtained through multilevel logistic regression model.

At first, a null model is considered (Model 1) to estimate community-level variance for justification of applicability of multilevel logistic regression model. Standard error of null model is 0.536 which gives an idea that there may have cluster effect and following the result the ICC and MOR arises. The intra-cluster correlation (ICC) value is estimated at 0.08 , which simply means that about 8 percent of the total variance in neonatal mortality in Bangladesh can be attributed to the communities in which the mothers were residing. This also implies that correlation between mothers living in the same community regarding the likelihood of experiencing neonatal mortality is 0.08 in studying that there exists cluster effect in neonatal mortality in Bangladesh. The estimated MOR is 1.67 which means that the likelihood of having neonatal mortality increased by 67 percent when a woman moved from a community with lower risk to a higher risk community. In other word it can also be said that the residual heterogeneity between communities increased by 1.7 times the individual odds of neonatal death when randomly picking out two mothers in different communities. Both ICC and MOR indicate that there exists community-level impact in neonatal mortality in Bangladesh.

In Model 2, individual level factors that are found significant in bivariate analysis are incorporated in null model. It is found that education level of father, delivery by caesarian section, PNC check time and breastfeeding has significant fixed effect on neonatal death. In Model 3, only community level factors are included. All community level factors that are found significant in bivariate analysis except division are considered. Although divisions show significant result in bivariate section, it is not considered in multivariate analysis as clusters are coincides in division and so the cluster effect can be nullified because of division $^{14}$. Among all community level factors, only telephone service is found to have significant adjusted impact on neonatal death. In both of Model 2 and Model 3, standard error for random part leads to existence of cluster impact on neonatal death (.573 and 0.449 respectively). Additionally, the estimated intra-cluster correlation (ICC) for each model is greater than zero (0.091 and 0.058 respectively) and the values of MOR for them are greater than 1 (1.727 and 1.537 respectively) that evidenced the cluster effect on neonatal death.

In Model 4, both individual and community level variables that are found to be significant in bivariate analysis (except division as it may nullify the cluster effect on neonatal mortality) are incorporated as independent variables.

Model 4 reveals that father's education has significant impact on neonatal death. The estimated value of odds ratio (OR) decreases as level of father's education increases that 
indicates that risk of neonatal death slows down with the improvement in father's education level.

The odds ratio for delivery by caesarean section is 0.71 which reveals that the odds to neonatal death for who has been delivered by caesarean section is 0.71 times of the odds to neonatal death for who has vaginal delivery. The results show that the odds ratio is 1.53 for qualified birth attendant that leads to conclusion that the odds of neonatal death for qualified birth attendant is 1.53 times of unqualified birth attendant. It is notable that the odds ratio for PNC within two days in model 4 is 0.48 indicating that the odds of neonatal death who took PNC in first two days is 0.48 times of odds of neonatal death who did not take PNC in first two days keeping all other factors at a fixed level. The estimated odds ratio for breast feeding $(\mathrm{OR}=0.015)$ discloses that the odds of neonatal death is lower if baby got breast milk immediately or within an hour compared to who did not get in within 1 hour.

Table 2. Fixed effects of individual and community level factors and random effects on neonatal mortality obtained from multilevel logistics regression model

\begin{tabular}{|c|c|c|c|c|c|c|c|c|c|}
\hline \multirow{2}{*}{$\begin{array}{l}\text { Variables and } \\
\text { Categories }\end{array}$} & \multicolumn{3}{|c|}{ Model 1} & \multicolumn{2}{|c|}{ Model 2} & \multicolumn{2}{|c|}{ Model 3} & \multicolumn{2}{|c|}{ Model 4} \\
\hline & OR & S.E & p-value & OR & p-value & OR & p-value & OR & p-value \\
\hline Intercept & 0.025 & 0.153 & $<0.001$ & 0.021 & $<0.001$ & 0.044 & $<0.001$ & 0.041 & $<0.001$ \\
\hline \multirow{2}{*}{\multicolumn{10}{|c|}{$\begin{array}{l}\text { Fixed Effects for Individual Level Variables } \\
\text { Education level of mother }\end{array}$}} \\
\hline & & & & & & & & & \\
\hline \multicolumn{10}{|l|}{ No Education / Primary } \\
\hline Secondary & & & & 1.463 & 0.445 & & & 1.385 & 0.512 \\
\hline Higher (ref.) & & & & - & & & & - & \\
\hline \multicolumn{10}{|l|}{ Education level of father } \\
\hline No Education/Primary & & & & 2.574 & 0.051 & & & 2.511 & 0.057 \\
\hline Secondary & & & & 2.540 & 0.046 & & & 2.482 & 0.052 \\
\hline Higher (ref.) & & & & - & & & & - & \\
\hline \multicolumn{10}{|l|}{ Wealth index } \\
\hline Middle/Poor & & & & 0.820 & 0.370 & & & 0.706 & 0.129 \\
\hline Rich (ref.) & & & & - & & & & - & \\
\hline \multicolumn{10}{|c|}{ Delivery by caesarean section } \\
\hline Yes & & & & 0.566 & 0.060 & & & 0.553 & 0.051 \\
\hline No(ref.) & & & & - & & & & - & \\
\hline \multicolumn{10}{|l|}{ Delivery assistance } \\
\hline Qualified & & & & 1.468 & 0.123 & & & 1.531 & 0.088 \\
\hline Unqualified (ref.) & & & & - & & & & - & \\
\hline \multicolumn{10}{|l|}{ PNC check time } \\
\hline First Two Days & & & & 0.453 & 0.001 & & & 0.480 & 0.002 \\
\hline Others (ref.) & & & & - & & & & - & \\
\hline \multicolumn{10}{|l|}{ PNC by qualified person } \\
\hline Yes & & & & 0.884 & 0.663 & & & 0.859 & 0.590 \\
\hline No(ref.) & & & & - & & & & - & \\
\hline \multicolumn{10}{|l|}{ Breastfeeding } \\
\hline Within 1 hour & & & & 0.015 & $<0.001$ & & & 0.015 & $<0.001$ \\
\hline Later (ref.) & & & & - & & & & - & \\
\hline \multicolumn{10}{|c|}{ Fixed Effect for community level variables } \\
\hline \multicolumn{10}{|c|}{ Telephone service availability } \\
\hline Yes & & & & & & 0.648 & 0.024 & 0.666 & 0.044 \\
\hline No(ref.) & & & & & & - & & - & \\
\hline \multicolumn{10}{|l|}{ Availability of FP methods } \\
\hline Yes & & & & & & 0.794 & 0.250 & 0.812 & 0.320 \\
\hline No(ref.) & & & & & & - & & - & \\
\hline \multicolumn{10}{|l|}{ Presence of pharmacy } \\
\hline Yes & & & & & & 0.847 & 0.421 & 0.764 & 0.213 \\
\hline No(ref.) & & & & & & - & & - & \\
\hline \multicolumn{10}{|c|}{ Presence of qualified doctor } \\
\hline Yes & & & & & & 0.879 & 0.535 & 0.906 & 0.652 \\
\hline No(ref.) & & & & & & - & & - & \\
\hline
\end{tabular}


Table 2 continued...

\begin{tabular}{l} 
Random Effect \\
\hline S. E \\
ICC \\
MOR \\
Model Statistic \\
AIC 1.636 \\
\hline Among the community level variables incorporated in \\
Model 4, telephone service has significant impact on \\
neonatal mortality (p-value=0.044). The odds of neonatal \\
death for those who belongs to a community where \\
telephone access is available is 0.67 times of odds of \\
neonatal death for those who does not belong to a \\
community where telephone access is found (OR=0.67). \\
While judging random effect of Model 4 , it is noticed that \\
standard error of full model is 0.5 which gives an idea that \\
there may have cluster effect both adjusting both individual \\
and community level variables. The intra-cluster correlation \\
(ICC) value is estimated at 0.07 and the estimated MOR is \\
1.612 which expose that the total variation in neonatal \\
mortality is attributable to variation in cluster even after \\
incorporating individual and community level variables in \\
the regression model.
\end{tabular}

While focusing on the Akaike Information Criteria (AIC), it is seen that null model possessed highest AIC value $(\mathrm{AIC}=1222.14)$ whereas $\mathrm{AIC}$ value is least for Model 4 $(\mathrm{AIC}=1037.080)$ which implies that factors are explained in the best way when we consider both individual and community level variables as covariates in the model (Model 4).

\section{Discussion}

Bangladesh has made tremendous progress in improving the health of its children and is one of the few countries in the developing world that is on track to achieve Millennium Development Goal 4. Despite a sharp decline in neonatal mortality rate, some burning issues still obstruct the pace of the current situation. In the remote areas of Bangladesh, the proneness of neonatal and infant mortality is still high. In this study, one of the main objectives is to analyze the cluster effect on neonatal mortality along with determining the factors that influence neonatal mortality.

The findings of this study shed light on the fact that the composition of community level characteristics influence the risk of neonatal mortality in conjunction with the individual level characteristics which is evident from previous study ${ }^{1}$. People living in same community will share same demographic and socio-economic condition which may lead to similar health outcome. That is why people living in same communities which suffer from deprivation of health care facilities and coexistence of poverty, illiteracy and unemployment are tend to be at higher risk of neonatal death due to cluster effect.

Among individual level factors, results shows that paternal education works as a protective factor for neonatal mortality rate which embraces the previous study ${ }^{15}$. The

\begin{tabular}{lll}
0.573 & 0.449 & 0.501 \\
0.091 & 0.058 & 0.071 \\
1.727 & 1.537 & 1.612 \\
\hline
\end{tabular}

$1040.67 \quad 1216.93 \quad 1037.080$

increasing level of education of father will improve the socio-economic status of household which can ensure the access to health care facilities that reduce neonatal mortality ${ }^{16}$. Moreover, it is found from the study that the rate of neonatal mortality is low for the women who had delivery by caesarean section ${ }^{17}$. Caesarean section performed with adequate medical facilities can assist to avoid the pregnancy complications and neonatal morbidity successfully that results in reduction in neonatal mortality rate. Trained birth attendance and PNC check time in first two days reduce neonatal mortality to a great extent that is supported by literature review ${ }^{9}$. The postnatal period is critical phase for both mother and newborn. Early postnatal care can identify the emergency problems and need of the newborn and suggestions by trained health professional during receiving PNC check time work as a shield against neonatal mortality. It is evident from the study that early initiation of breastfeeding lowers the risk of neonatal mortality $^{1,18}$. Breast milk contains colostrum in first stage which is high in antibodies. If babies are breastfed early, the antibody of colostrum passes from mother to babies that strengthen their immune system against infectious diseases and as a result, neonatal mortality decreases. Additionally, the communities that have available accessibility of telephone service experience lower risk of neonatal mortality as they can call to health care center in case of emergency.

The discussion of findings of this study demonstrates that both individual and community level factors influence neonatal mortality. In the context of patriarchal society in Bangladesh, it is necessary to ensure that the education on mother and child health care should also be given to father along with mother to reduce neonatal mortality. It should be taken care of that health care facilities like hospital facility, qualified delivery assistance etc. should be provided to all over Bangladesh. Every mother should take postnatal care after delivery. It is highly recommended that the PNC must be received by qualified person. It helps to reduce the chance of child death. Government and non-governmental bodies should implement programs on promotion of exclusive breastfeeding. Moreover, the communities that are at high risk of neonatal death should be provided basic needs like qualified doctor facility, pharmacy facility, proper telephone service etc. that may protect the neonates from vulnerability of untimed death.

However, this study can be conducted on three level: Division level, Community level and Individual level to examine all the factor. If there exists any division level random effect for which the rate of neonatal mortality has significant change, the study will have further scope. 


\section{Acknowledgement}

The author is thankful to National Institute of Population Research and Training (NIPORT), Bangladesh for providing Bangladesh Demographic and Health Survey 2014 data.

\section{References}

1. Kayode, G.A., E. Ansah, I.A. Agyepong, M. AmoakohColeman, D.E. Grobbee, and K. Klipstein-Grobusch, 2014. Individual and Community Determinants of Neonatal Mortality in Ghana: A Multilevel Analysis. BMC pregnancy and childbirth, 14(1), 165.

2. National Institute of Population Research and Training (NIPORT), Mitra and Associates, and ICF International. Bangladesh Demographic and Health Survey 2014. Dhaka, Bangladesh and Calverton, Maryland, USA: Mitra and Associates, and ICF International, 2016.

3. UNICEF DATA. (n.d.). Neonatal mortality - UNICEF DATA. [online] Available at: https://data.unicef.org/topic/ child-survival/neonatal-mortality/ [Accessed 10 May 2019].

4. World Health Organization. (n.d.). Success Factors for Women's and Children's Health: Multisector Pathways to Progress. [online] Available at: https://www.who.int /pmnch/ successfactors/en/ [Accessed 10 May 2019].

5. World Health Organization. (n.d.). Sustainable Development Goal 3: Health. [online] Available at: https://www.who.int /topics/sustainable-development-goals/targets/en/ [Accessed 10 November, 2019].

6. General Economics Division (GED), Planning Commission Government of the People's Republic of Bangladesh. Millennium Development Goals: Bangladesh Progress Report 2015, September, 2015.

7. HPNSDP (Health, Population and Nutrition Sector Development Program, 2011-2016), PIP (Program Implementation Plan), July 2011. Volume-1, Planning Wing, Ministry of Health and Family Welfare, Government of the People's Republic of Bangladesh.

8. Neupane, S. and D.T. Doku, 2014. Neonatal mortality in Nepal: a multilevel analysis of a nationally representative. Journal of epidemiology and global health, 4(3), 213-222.
9. Titaley, C.R., M.J. Dibley, K. Agho, C.L. Roberts and J. Hall, 2008. Determinants of neonatal mortality in Indonesia. BMC public health, 8(1), 232.

10. UNICEF DATA. (n.d.). Newborn care - UNICEF DATA. [online] Available at: https://data.unicef.org/topic/maternalhealth/newborn-care/ [Accessed 10 May 2019].

11. Goldstein, H., 2010. Multilevel Statistical Models. New York: Wiley Series in Probability and Statistics.

12. Gelman, A. and J. Hill, 2006. Data Analysis Using Regression and Multilevel Hierarchical Models. Cambridge university press.

13. Merlo J, B., H. Chaix , Ohlsson, et al, 2006. A Brief Conceptual Tutorial of Multilevel Analysis in Social Epidemiology: Using Measures of Clustering in Multilevel Logistic Regression to Investigate Contextual Phenomena. Journal of Epidemiology \& Community Health, 60, 290297.

14. Mahmood, S., B. Zainab, and A.M. Latif, 2013. Frailty Modeling for Clustered Survival Data: An Application to Birth Interval in Bangladesh. Journal of Applied Statistics, 40(12), 2670-2680.

15. Al Kibria, G.M., V. Burrowes, A. Choudhury, A. Sharmeen, S. Ghosh, A. Mahmud and K.C. Angela, 2018. Determinants of Early Neonatal Mortality in Afghanistan: An Analysis of the Demographic and Health Survey 2015. Globalization and Health, 14(1), 47.

16. Choudhury, P.K., 2015. Explaining the Role of Parental Education in the Regional Variations in Infant Mortality in India. Asia \& Pacific Policy Studies, 2(3), 544-572.

17. Ghaedmohammadi, Z., A. Anaraki, A. Khajeian, M. Khajehian and A. Ostovar, 2015. Association of Caesarean Section and Neonatal Death: a Population-based CaseControl Study in Islamic Republic of Iran. EMHJ-Eastern Mediterranean Health Journal, 21(4), 266-272.

18. Phukan, D., M. Ranjan, and L.K. Dwivedi, 2018. Impact of Timing of Breastfeeding Initiation on neonatal mortality in India. International breastfeeding journal, 13(1), p.27. 
